\title{
VoD Service Traffic Monitoring with Neural Agent
}

\author{
Danielo G. Gomes ${ }^{1 *}$, Nazim Agoulmine ${ }^{1}$, and J.N. de Souza ${ }^{2}$ \\ ${ }^{1}$ Laboratory of Complex Systems - LSC, University of Evry, \\ 40, Rue du Pelvoux - CE 1455 Courcouronnes 91020 Evry Cedex, France \\ \{dgomes,nazim\}@iup.univ-evry.fr \\ 2 Teleinformatics Engineering Department - DETI, Federal University of Ceará - UFC, \\ Campus do Pici - Bloco 705, 60455-760, Fortaleza-CE, Brazil \\ neuman@ufc.br
}

\begin{abstract}
Video and LAN traffics can be modeled as self-similar processes and the Hurst parameter is a measure of the self-similarity of a process. The purpose of this work is to use this characteristic of Internet traffic allowing future Video on Demand Service Providers (VDSPs) to optimise their bandwidth utilization and consequently their communications cost. The work refers to one aspect of a global project that specifies intelligent agent architecture to manage the relationship among VDSP, Internet Service Providers (ISPs) and end-customers. In this paper, we also discuss the egress traffic aspect of the VDSP and propose a neural network approach to monitor and to estimate the nature of the future Value Added Service Provider (VASP) egress traffic using the Hurst parameter. This approach takes into account the real MPEG (Moving Picture Experts Group) streams in two scenarios: (i) with 13 individual MPEG streams; and (ii) with 6 mixed sequences.
\end{abstract}

\section{Introduction}

With the explosive growth of the Internet and of private networks related to it, a large number of new demands have increased. Low-volume Telnet conversations have been replaced by high-volume Web traffic, which it has been increasing the graphic intensity. Moreover, real-time applications have demanded an even quicker network response. In order to deal with these demands, we need nor only to make Internet capacity bigger but also to adopt accurate methods for managing the traffic. Several studies have claimed that different types of network can be accurately modelled using a self-similar process, i.e., a process capable to capture the Long-Range Dependence (LRD) phenomenon exhibited by such traffic. Furthermore, studies have demonstrated that the long range dependencies may have a pervasive effect on queuing performance. In fact, there is clear evidence that it can potentially cause massive cell losses. Such queuing system suffers from the buffer inefficacy phenomenon. The solution proposed

\footnotetext{
* This work is supported by CAPES (Brazil) under PhD Fellowship number 266/99-I.
}

A. Marshall and N. Agoulmine: MMNS 2003, LNCS 2839, pp. 516-528, 2003.

(C) IFIP International Federation for Information Processing 2003 
by increasing the buffer size is not significantly effective for decreasing the buffer overflow probability [1].

This paper deals with the future Value Added Service Providers (VASPs), especially video on demand (VoD) VASP or VDSP, which is part of the work undertaken at the LSC Lab. This research goal is to define a global architecture based on intelligent agents whose intelligence derives from neural networks. The aim of that architecture is to allow Video on Demand Service Providers (VDSPs) to improve the use of the bandwidth as well as reduce the risk of the degradation of the Quality of Service (QoS). VDSPs are connected to a particular Internet Service Provider (ISP) that provides the necessary bandwidth that permit to end customers to access video service. The egress traffic from the VDSP domain is composed by different video streams which are sent to the end customers. The classical approach for VDSP to allow this communication is to establish a contract with an ISP that provides it with a certain bandwidth. However, the main problem with this approach is that the bandwidth is usually provided as leased line. Then, if the sum of traffic is inferior to the available bandwidth, the VDSP will pay for bandwidth without using it. On the other hand, if the number of customers increases, the allocated bandwidth may not be enough to keep the QoS at an acceptable level and the VDSP will have to refuse new connections. Thus, it is important for the VDSP to dynamically control the ISP allocated bandwidth to either increase or decreasing it according to the forecasting traffic. Since the Internet exhibit a long-range dependence, one possible approach to predict the traffic is to calculate in real time the nature of the VDSP egress traffic and detect whether it is possible or not to predict what the future traffic will look like. If the traffic significantly decreases, it is the time for the VDSP to negotiate on line a decrease of its bandwidth reservation. On the other hand, if the traffic goes over the maximum bandwidth reservation, the VDSP will negotiate an increase of its bandwidth. In the other cases, either the bandwidth allocation is optimal, or it is not possible to have a clear view of the future.

The Hurst parameter or parameter $H$ characterizes the self-similarity process degree. The real-time calculation of this parameter will give a good view of the traffic on a particular link. The objective of this paper is identify the nature of the VDSP egress traffic by calculating the Hurst Parameter based on monitoring of traffic at the VDSP access router. In this paper, we evaluate the possibility to use a Neural Network (NN) to estimate in real time the Hurst parameter value. This neural network is managed by a agent that is placed in or near the VDSP access router and capable to take a decision regarding forecasting bandwidth reservation. The traffic for this analysis is composed of MPEG (Moving Picture Experts Group) real traces. Results indicate that the neurocomputation approach provides reasonably accurate results and is proper for real-time implementation.

The remainder of this paper is organized as follows. Section 2 provides some notions about Internet traffic, Hurst parameter, agent technology and neural networks. The proposed architecture is presented in Section 3, the MPEG streams used in Section 4 and the neural estimator in Section 5. Numerical results are shown in Section 6 and the Section 7 concludes the paper. 


\section{Background}

\subsection{Internet Traffic}

The Internet Protocol (IP) is part of the TCP (Transmission Control Protocol)/IP protocol suite and is the most widely-used internetworking protocol. The IP function is to transfer data blocks, namely datagrams, transported from the source host to the destination host.

IP is a connectionless network protocol. Every IP datagram is seen as an independent unit. The communication is unreliable, i.e., there is no end-to-end recognition nor between intermediate nodes. In addition, there is no mechanism of error control of the data transmitted and no flow control is used.

In the old days, most of the data carried on networks was textual data. Today, with the rise of multimedia applications and network technologies, multimedia has become an indispensable feature on the Internet. Real-time voice and video applications become more and more popular on the Internet.

However, multimedia networking is not a simple task and, so far, the Internet has been following the best-effort delivery model. There is no admission control and the network makes its best to transmit information as quickly as possible but no assurance can be guaranteed about the delivery of the packets. Most of the applications on the Internet were elastic in nature, in that they tolerated packet delays and packet losses and they could be relatively served by the best-effort model. Nevertheless, the emerging multimedia traffic, such as voice and video, do not perform well under extreme variations in the delay and excessive dropping of packets.

In the current Internet model, the inelastic traffic does not perform adequately and interferes to the elastic traffic, leaving it with less bandwidth. To adapt inelastic traffic, it is necessary extending the Internet model to support the newer applications providing QoS control [2],[3].

\subsection{The Hurst Parameter}

Let $x(t)$, with $t=0,1,2, \ldots$, a stationary stochastic process [4]. For each $m=1,2, \ldots$, let $x^{(m)}(k), k=1,2,3, \ldots$, denote a new series obtained by averaging the original series $x(t)$ over non-overlapping blocks of size $m$.

A process $X$ is called exactly second-order self-similar with parameter $H=1-\beta / 2,0<$ $\beta<1$, if its autocorrelation function is [4]:

$$
r^{(m)}(k)=\frac{1}{2}\left[(k+1)^{2-\beta}-2 k^{2-\beta}+(k-1)^{2-\beta}\right] \equiv g(k), 0<\beta<1, k=1,2, \ldots
$$

and $X$ is called asymptotically second-order self-similar with parameter $H=1-\beta / 2$, $0<\beta<1$, if for all $k=1,2, \ldots$,

$$
\lim _{m \rightarrow \infty} r^{(m)}(k)=\frac{1}{2}\left[(k+1)^{2-\beta}-2 k^{2-\beta}+(k-1)^{2-\beta}\right] \equiv y(k)
$$


In self-similar processes, the autocorrelations decay hyperbolically implying in a nonsummable autocorrelation function $\Sigma_{k} r(k)=\infty$ (long-range dependences),

The Hurst parameter $(H)$ gives the degree of self-similarity of a process, and, consequently, expresses the pattern of dependencies of a process. If $0.5<H<1$, the process is a Long-Range Dependent (LRD) process. If $0<H<0.5$ it is an anti-persistence process, and if $H=0.5$ it is a Short-Range Dependent (SRD) process. Fig. 1 illustrates the auto-correlation decay for different values of the Hurst parameter.

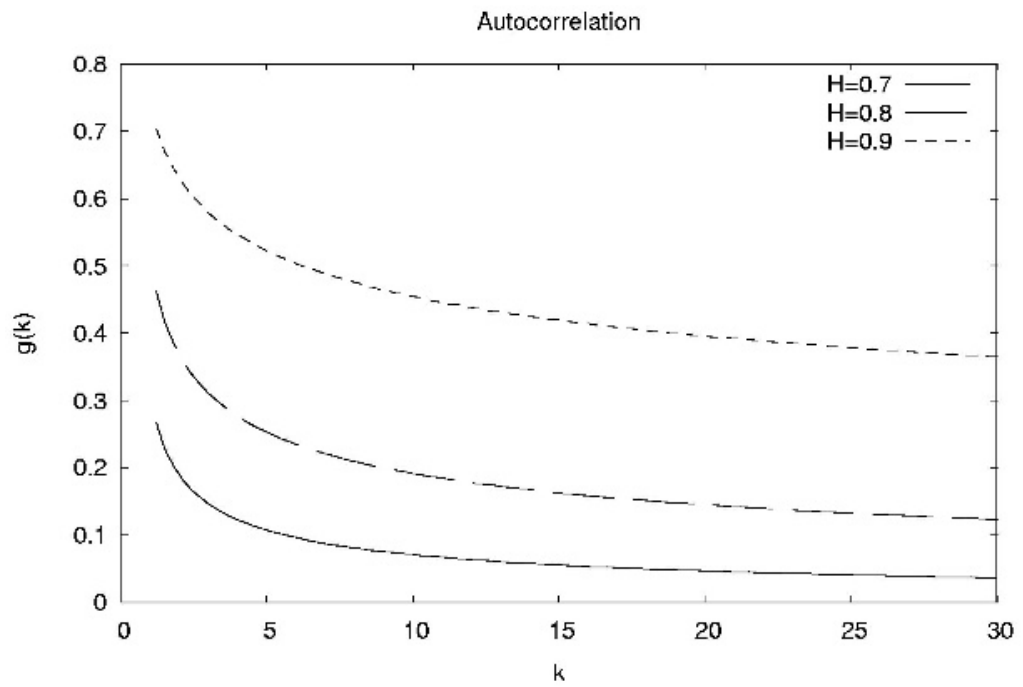

Fig. 1. Autocorrelation function (1) of an exact second-order self-similar with parameter $H=1-\beta / 2$

\subsection{Agent Technology}

The agent concept has been widely proposed and adopted within both the telecommunications and Internet communities. Agent is a key tool in the creation of an open, heterogeneous and programmable network environment. This trend is motivated by the desire of using agents to solve some problems encountered in large scale distributed and real-time systems such as the volume and complexity of the tasks, latency, delays, and others. Generally, an agent can be faced as an assistant or helper, which performs routine and complicated tasks on the user's behalf. In the context of distributed computing, an agent is an autonomous software component that acts asynchronously on the user's behalf. Agent types can be broadly categorized as static or mobile. The majority of current communication system architectures employ the client-server method, which requires multiple transactions before a given task can be accomplished. This can lead to increased signalling traffic throughout the network. This problem can 
rapidly escalate in an open network environment that spans multiple domains. As a solution, mobile agents can move among computers and interact with other agents. For example, mobile agents can be delegated to complete specific tasks on their own, providing that a certain set of constraints or rules have been defined for them [5]. They can be dispatched across the network in the form of mobile program or mobile code that can be recompiled and executed in the remote host.

The main motivation of the use of agent technology herein is driven by the desire to automate the control and management processes by allowing for more programmability of the network to customize the provision of new information and telecommunication services Agents can also be used to implement Service Level Agreements (SLAs) between different actors of the network and service era. Agent can then be used as brokers or mediators between end users and a service provider in order to implement the SLA. In this way, complicated QoS metrics (from end user's point of view) can be communicated in a simplified manner. Service provider and network provider agents can then negotiate with users' agents to provide required service [6].

\subsection{Neural Networks}

The Neural Networks (NNs) appeared as an attempt of overcoming the sequential computers, based on the parallel processor structures, which can adapt the answer to the experience (training). A neural network attempts to emulate the way a human brain works.

A neural network is a system formed by a high number of simple processors (neurons or nodes), highly interconnected and based on a simplified model of the neuron. Neurocomputation is a computational approach of the neural networks for the processing of the information [7].

In fact, the weights represent the knowledge of the $\mathrm{NN}$ at the end of the training process and the learning is the result of all the process. Therefore, the learning is a process where the synaptic connections of the neural network are adapted by a continuous stimulus process from the environment where the network is inserted [8].

\section{Proposed Architecture}

The target architecture we intend to deploy is composed by a set of agents. In order to be operational, we suppose that all actors (VDSP, ISP and the end customer) will deploy an agent platform. At the ISP boundary, the platform will support four types of agents: a Policy agent responsible for the management of global ISP policies that defines high level specification of the system behaviour (pricing rules, allocation rules, etc), a Reservation agent responsible to interacting with physical equipments (routers) configuring them to provide the bandwidth agreed at the business level to the ISP customers (end customer and VDSP), an Accounting and Billing Agent responsible to accounting and billing network resource usage and allocating them to ISP customers 
and finally the Bandwidth Broker Agents responsible to interacting with ISP customer with the purpose of negotiating bandwidth allocation (Fig. 2).

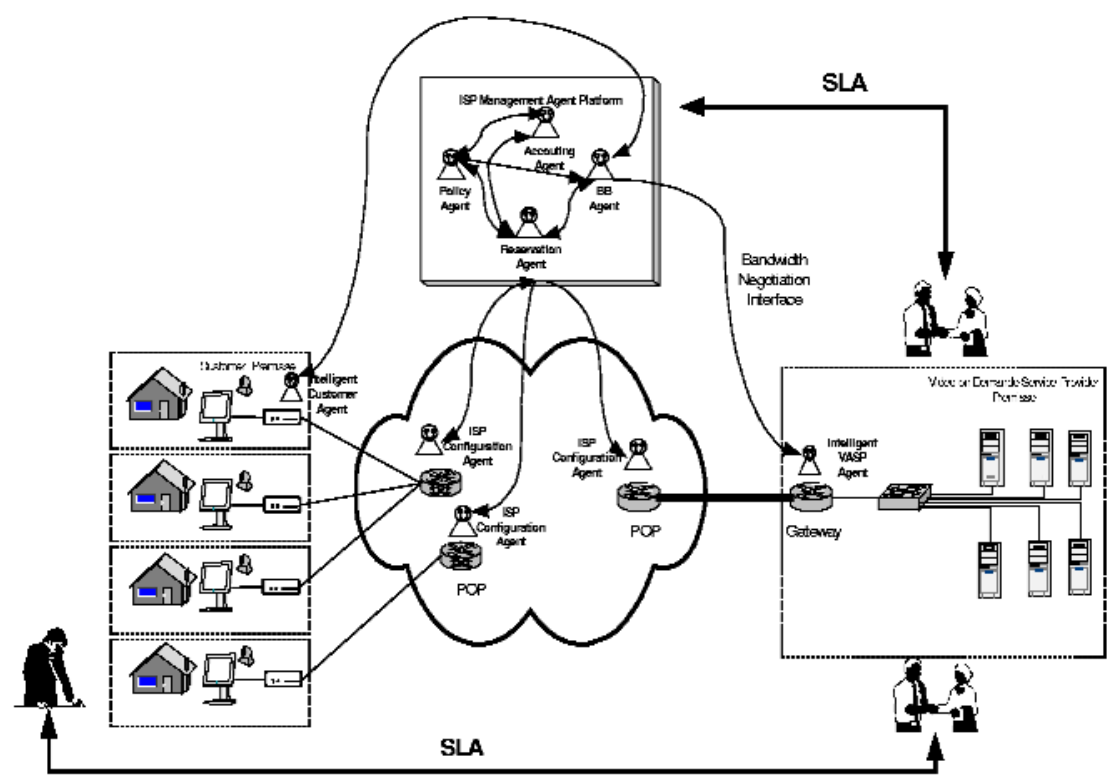

Fig. 2. Multi-agents environment

The VDSP intelligent agent is responsible for the monitoring of the egress traffic and the prediction of the forecasting traffic. At the end customers' premise, a negotiation agent allows the end customer to register at the $\mathrm{VoD}$ service. Also it is responsible for the end customer bandwidth availability.

The starting point of the process is a VDSP willing to offer a VoD service to a large number of end users. The VASP has established an agreement with an ISP to provide connectivity with a predefined bandwidth to end customers. We initially consider that end customers have already agreed an IP service with the same ISP using DSL technology. We suppose that the ISP is able to control the bandwidth allocated to each end user. Each customer can on-line register with the VoD service in order to access a particular movie stream at a certain date/time from the movie portfolio provided by the VDSP. Due to the bursty nature of the traffic that is sent by the video servers, the interest of the VDSP is to dynamically adapt its bandwidth reservation to a level compatible with the global traffic avoiding under provisioning or over provisioning and extra cost or quality of service degradation (Fig. 3 shows an example).

This approach is only possible if the VDSP is able to predict which traffic will appear in the middle term time scale. This task is assigned to the Intelligent VASP agent. Once the agent detects an important change in the traffic, it informs the BB agent of 


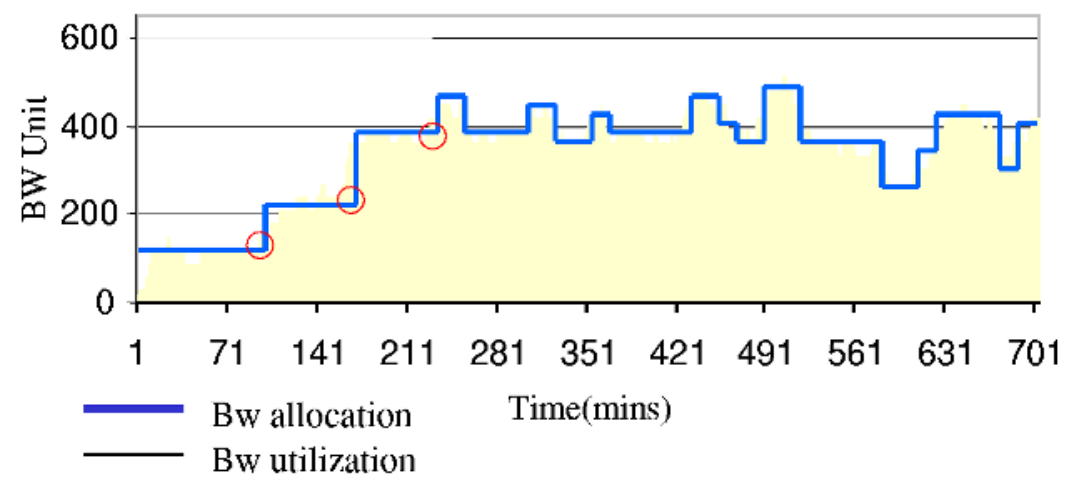

Fig. 3. Example of efficient bandwidth allocation

the ISP management system in order to request or release resources adapting to the new traffic profile. Therefore, the Intelligent VDSP Agent is at the heart of the suggested architecture.

In this context, we aim to investigate the possibility to predict the nature of the traffic so that to permit to renegotiate the allocated bandwidth. The renegotiation dialog is performed between the intelligent VDSP and the ISP BB agents (Fig. 4).

This aspect of the architecture and the internal interactions among the agents are not specified in this paper. Only the intelligent VDSP behaviour is mentioned. So the objective is using a neural network that allows the VDSP agent to take a decision regarding near future. Idea is to train the neural network with existing traffic profile and determine whether it is capable to calculate the Hurst parameter that gives information about the nature of the traffic in a relatively short period. In the real situation, it is possible to affirm that we can train the neural network including the existing video streams as the VDSP knows them initially. Every time a new movie is included in the movie portfolio, the agent is trained with its traffic.

\section{MPEG Streams}

Several coding algorithms for the compression of these streams were developed due to the high bandwidth needs of uncompressed video data streams. At the moment, the MPEG coding scheme is widely used for any type of video applications. Table 1 shows the MPEG sequences ${ }^{1}$ used for Hurst parameter estimation as well as their respective $\mathrm{H}$-values estimated by the $\mathrm{R} / \mathrm{S}$ method [1] and the sequences were MPEG-1 encoded with sampling rate of 25 frames/sec .

1 These traces were generated by $\mathrm{O}$. Rose and are available in http://www3.informatik.uniwuerzburg.de/ rose/. 


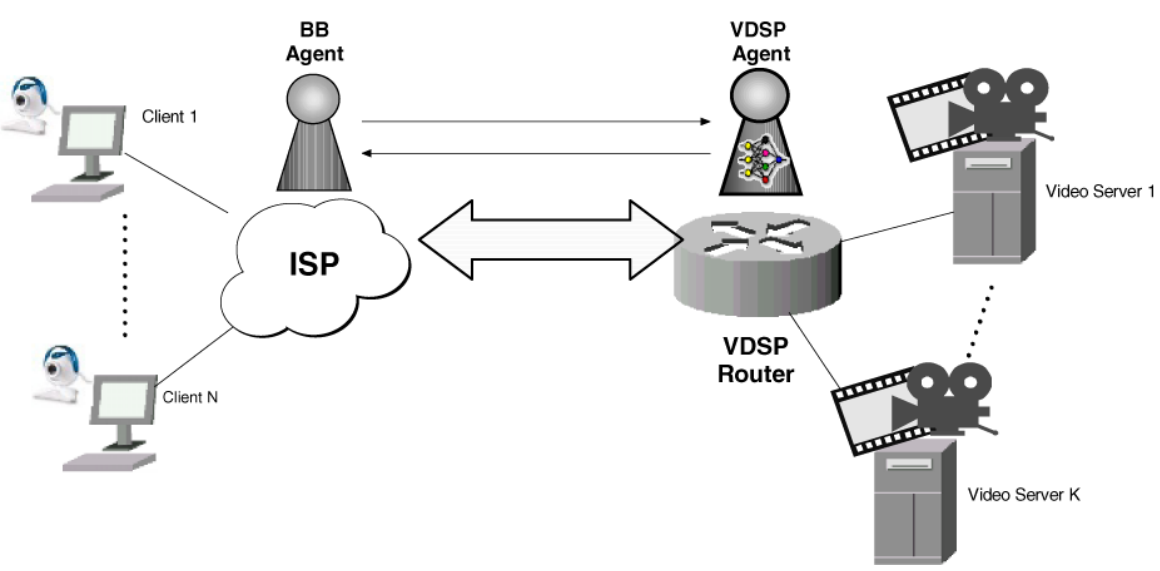

Fig. 4. VoD Model

It is known that in the event of video traffic a larger $\mathrm{H}$-value reflects a larger amount of movement in the video sequence [9]. Note in Table I that all sequences have $\mathrm{H}$ values which are higher than 0.73 , therefore, the existence of long-range dependencies can be assumed.

Table 1. Hurst parameter of the Encoded Sequences

\begin{tabular}{|c|c|}
\hline Sequence & Parameter H \\
\hline race & 0.99 \\
\hline soccer & 0.91 \\
\hline lambs & 0.89 \\
\hline terminator & 0.89 \\
\hline mtv & 0.89 \\
\hline simpsons & 0.89 \\
\hline talk & 0.89 \\
\hline dino & 0.88 \\
\hline atp & 0.88 \\
\hline mr.bean & 0.85 \\
\hline Asterix & 0.81 \\
\hline news & 0.79 \\
\hline starwars & 0.74 \\
\hline
\end{tabular}

\section{The Neural Agent Estimator}

In this work, we have used a feed-forward network architecture with a backpropagation momentum training algorithm to estimate the parameter $\mathrm{H}$ of MPEG streams. The back-propagation algorithm was considered provided that it is the most 
successful algorithm for the design of multilayer feedforward networks. The number of neurons in the input-output layers was defined according to the structure of the problem. The output variable is the parameter $H$, i.e., the neural network presents one neuron on the output layer. There is not established procedure of choice for the optimum number of neuron. Then, experiments were tried with 2,5,10, 15 and 20 neurons and the better results were those obtained with 15 hidden neurons. Hence, the neural network has 10 input neurons, 15 hidden neurons and 1 output neuron. Fig. 5 shows the neural topology used and the results were derived by using the JavaNN Simulator [10].

The NN used 50 patterns for each MPEG real sequence. Each pattern has 10 video frames (input) and its respective Hurst parameter (output). Below is the summary of the neural agent behaviour:

i. Train the VDSP agent with existing movies;

ii. Install agent in the access router (border router);

iii. During execution, the agent estimate the Hurst parameter.

The calculation of the Hurst parameter will benefit in the future the agent to estimate the forecast traffic and decide whether or not to request or reduce bandwidth.

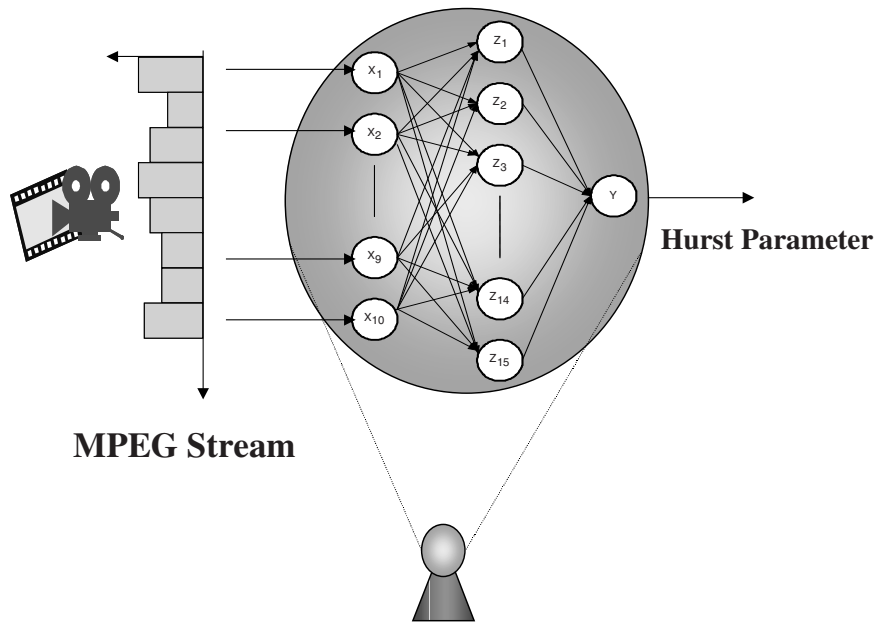

Fig. 5. Neural agent structure

\section{Results and Discussion}

The learning phase included simulations with logarithmic and sigmoid activation functions. Fig. 6 (a) and (b) show that the second case is better than the first given that it needs a smaller quantity of epochs. 
Activation Function: Logarithmic

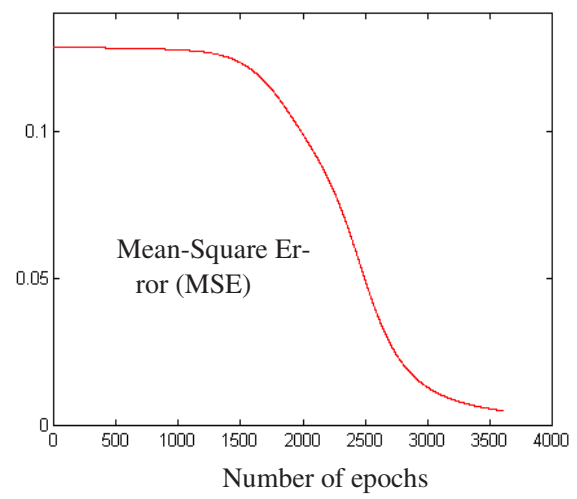

(a)
Activation Function: Sigmoid

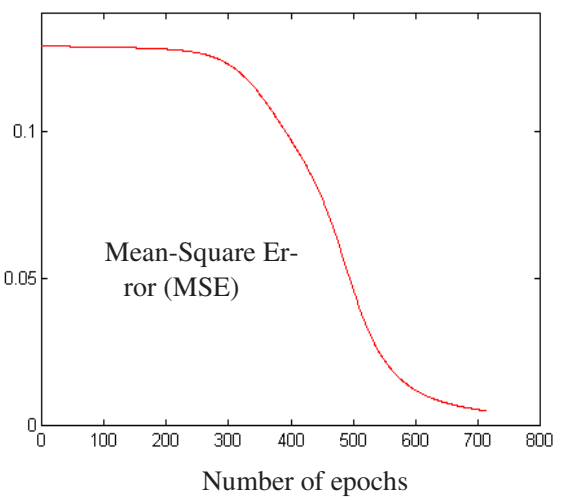

(b)

Fig. 6. Training error with (a) logistic and (b) sigmoid function

In the case of MPEG individual streams, the neural estimator error for different values of the parameter $\mathrm{H}$ is showed in Table 2 and in Fig. 7. Note that if the parameter is closer to 1 it means that the estimation error is smaller.

Table 2. H-estimation of individual streams

\begin{tabular}{|c|c|c|c|}
\hline Sequence \# & Parameter H & NN Estimation & Error \\
\hline 1.race & 0.99 & 0.96 & 0.03 \\
\hline 2.soccer & 0.91 & 0.79 & 0.12 \\
\hline 3. lambs & 0.89 & 0.70 & 0.19 \\
\hline 4. terminator & 0.89 & 0.71 & 0.18 \\
\hline 5. mtv & 0.89 & 0.69 & 0.20 \\
\hline 6. simpsons & 0.89 & 0.70 & 0.19 \\
\hline 7. talk & 0.89 & 0.71 & 0.18 \\
\hline 8. dino & 0.88 & 0.70 & 0.18 \\
\hline 9. atp & 0.88 & 0.68 & 0.20 \\
\hline 10. mr.bean & 0.85 & 0.65 & 0.20 \\
\hline 11. asterix & 0.81 & 0.6 & 0.21 \\
\hline 12. news & 0.79 & 0.6 & 0.19 \\
\hline 13. starwars & 0.74 & 0.55 & 0.19 \\
\hline
\end{tabular}

Note that in Fig. 7 that for values of the Hurst parameter close to 1 (LRD), the neural network estimator produces accurate results. However, the error increases and remains about in the same value for the rest of the sequence $(\sim 20 \%)$.

Afterwards, we mixed some sequences and estimated the Hurst parameter by the R/S and neural estimators, respectively (Table 3 and Fig. 8 show the results). 


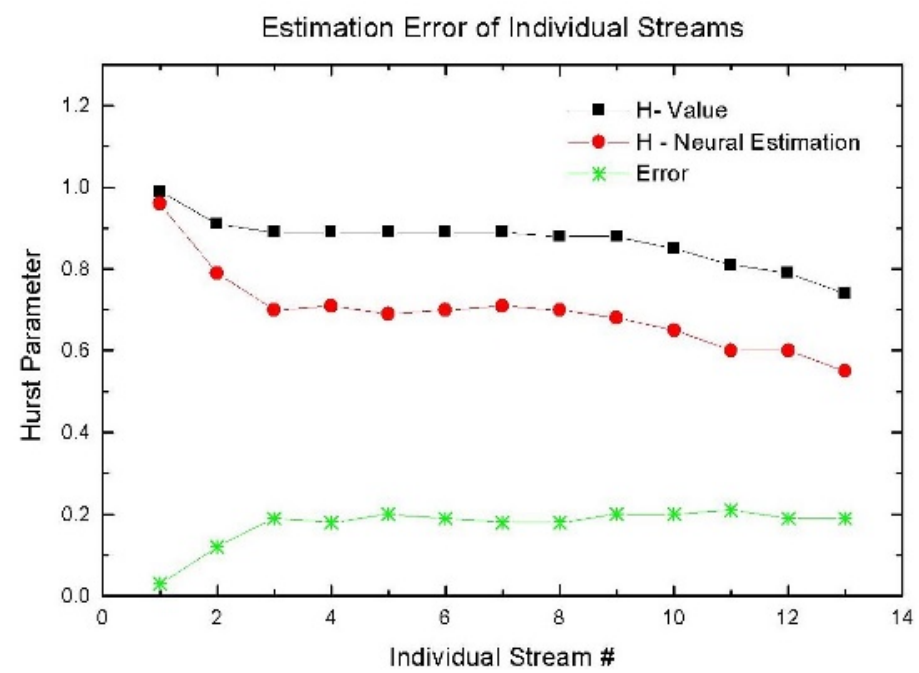

Fig. 7. Neural estimator error of the 13 individual streams

Table 3. H-estimation of mixed streams (6 sequences)

\begin{tabular}{|c|c|c|c|}
\hline Sequence \# & R/S Estimation & NN Estimation & $\begin{array}{c}\text { Difference } \\
(\mathbf{R} / \mathbf{S}-\mathbf{N N})\end{array}$ \\
\hline 1. race + news + starwars & 0.981 & 0.976 & 0.005 \\
\hline 2.soccer + asterix & 0.90 & 0.89 & 0.01 \\
\hline 3.lambs + mr.bean & 0.87 & 0.86 & 0.01 \\
\hline 4. terminator + atp & 0.88 & 0.895 & -0.015 \\
\hline 5.mtv + dino & 0.88 & 0.87 & 0.01 \\
\hline 6.simpsons + talk & 0.885 & 0.875 & 0.01 \\
\hline
\end{tabular}

Although error increase, the neural networks only demanded one hundreds frames for each MPEG sequence while each total trace has 40000 frames. The drawback of the neural networks is the delay in learning which impact, however, decreases for large traces.

\section{Conclusion}

The present work investigates the effectiveness of a neural network $\mathrm{H}$-estimator for VoD traffic monitoring. Neural networks, even demanding a significant time fortraining, represent an accurate and fast estimation of the parameter $H$. The presented approach aims to use this neural network as the intelligent part of an agent that allows a 


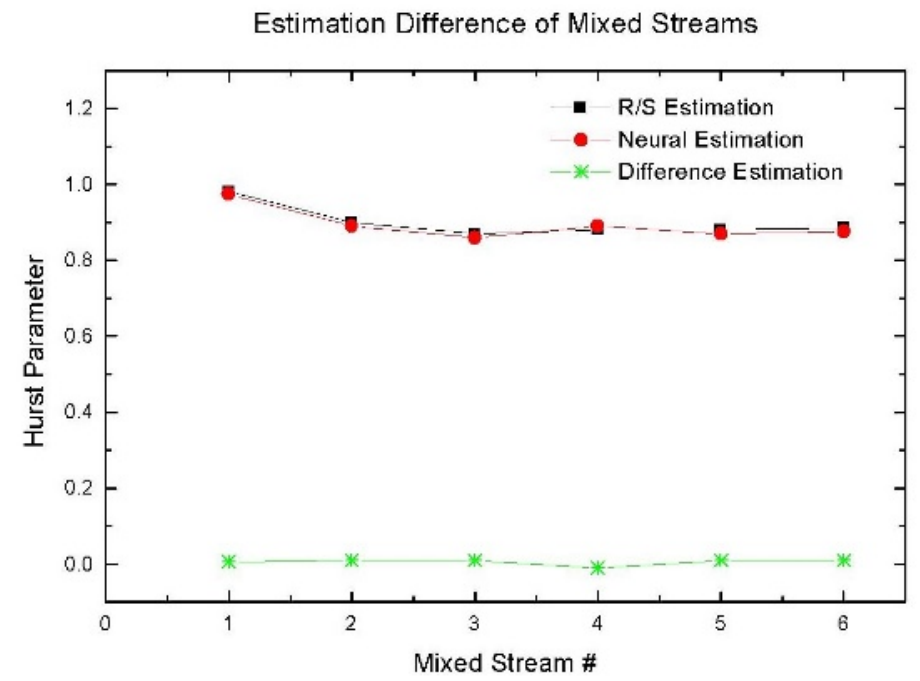

Fig. 8. R/S and neural H-estimations of the 6 mixed streams

VDSP to predict its traffic. The global architecture will permit in a competitive telecommunication market to an ISP to provide a new type of on-demand bandwidth provisioning. The numerical results showed that the neural estimation presents an error approximately up to $20 \%$ for the MPEG individual streams. However, concerning to the MPEG mixed streams, the difference between neural and R/S estimators is almost zero. Such results are considered interesting given that in $\mathrm{VoD}$ real scenario flow is aggregated.

\section{References}

1. Taqqu M., Teverovsky V., Willinger W.: Estimators for Long-Range Dependence: an Empirical Study. Fractals, vol 3, No 4 (1995) 785-788

2. Blake S., Black D., Carlson M., Davies E., Wang Z., Weiss W.: RFC 2475: An Architecture for Differentiated Services. Network Working Group, IETF, December 1998.

(www.ieft.org)

3. Braden R., Clark D., Shenker S.: RFC 1633: Integrated Services in the Internet Architecture: An Overview. ( July 1994). fttp://ftp.isi.edu/in-notes/rfc1633.ps

4. Leland W., Taqqu M., Willinger W., Wilson D.: On the Self-Similar Nature of Ethernet Traffic (Extended Version). IEEE/ACM Transaction on Networking, vol 2, no 1, (February 1994) $1-15$

5. Chieng D.: A Mobile Agent Brokering Environment for The Future Open Network Marketplace. Seventh International Conference On Intelligence in Services and Networks (IS\&N2000), Athens, Greece, Feb 2000 
6. Agoulmine N., Dragan D., Gringel T., Hall J., Rosa E., Tschichholz M.: Trouble Management for Multimedia Services in Multi-Provider Environments. International Journal on Network and Service Management, Wiley publisher, Vol nº ${ }^{\circ}$, (January 2000) 99-123

7. Hect-Nielsen R.: Neurocomputing. Addison-Wesley Publishing Company (1990)

8. Fausset L.: Fundamentals of Neural Networks. Prentice-Hall International, New Jersey, (1994)

9. Beran J., Sherman R., Taqqu M., Willinger W.: Variable-Bit-Rate Video Traffic and LongRange Dependence. IEEE Transactions on communications, (1994)

10. Stuttgart U., JNNS - Java Neural Network Simulator - User Manual, Version 1.0 beta http://www-ra.informatik.uni-tuebingen.de/downloads/JavaNNS/ 